\section{Dr. Jinno replies}

To the Editor:

We thank Dr. Mullins ${ }^{1}$ for his comments on our recent $\operatorname{article}^{2}$ on the trends in emergency department (ED) visits and charges for gout in the United States between 2006 and 2012. In our paper, we showed that the number of gout ED visits and cost significantly rose over the years.

Concerning the comment of Dr. Mullins on the possibility of the colchicine policy change affecting sharp increase of ED visits since 2009, it is beyond the data available in the Nationwide Emergency Department Sample (NEDS). We stated in the Discussion section that we could not perform patient-level analyses such as colchicine prescription across different insurance types because NEDS does not contain these data.

However, we acknowledge this as an important point. Recently, Kesselheim, et $\mathrm{al}^{3}$ showed a reduction in colchicine initiation and an increase in patient spending among patients with gout in United Health-affiliated enrollees after January 2011 when the market exclusively enforced colchicine patency. Along with our data, it is speculated that the reduction in colchicine use may have contributed to unnecessary office or hospital visits by patients because of suboptimal care. Further followup studies may be warranted to see whether healthcare use by patients with gout changes with expected improvement of patient access to affordable colchicine in coming years ${ }^{4}$.
SADAO JINNO, MD, MSc, Section of Rheumatology, Department of Medicine, Boston University School of Medicine, 650 Albany St., Suite 200, Boston, Massachusetts 02118, USA.E-mail: Sadao.jinno@bmc.org

\section{REFERENCES}

1. Mullins ME. Association of colchicine price and emergency department visits for gout. J Rheumatol 2017;44:397.

2. Jinno S, Hasegawa K, Neogi T, Goto T, Dubreuil M. Trends in emergency department visits and charges for gout in the United States between 2006 and 2012. J Rheumatol 2016;43:1589-92.

3. Kesselheim AS, Franklin JM, Kim SC, Seeger JD, Solomon DH. Reductions in use of colchicine after FDA enforcement of market exclusivity in a commercially insured population. J Gen Intern Med 2015;30:1633-8

4. American College of Rheumatology. ACR plays pivotal role in improving patient access to affordable colchicine. [Internet. Assessed December 16, 2016.] Available from: www.rheumatology.org/ About-Us/Newsroom/Press-Releases/ ID/30/ACR-Plays-Pivotal-Role-in-Improving-Patient-Access-toAffordable-Colchicine

J Rheumatol 2017;44:3; doi:10.3899/jrheum.161513 